\title{
EXOPLANETS
}

\section{Survival of the fattest}

Mon. Not. R. Astron. Soc. 493, 765-775 (2020)

All of the planets we know about orbit low-mass $\left(\lesssim 3.1 M_{\odot}\right)$ stars. Does that mean that planets around more massive stars do not exist? Since targeted searches of O- and B-type stars are challenging, Dimitri Veras and colleagues have taken a different approach. Could polluted massive white dwarfs (WDs) offer a clue to the planetary systems that once existed around their intermediate-mass progenitors?

Veras and collaborators use models to wind back the clock from the end-state WDs to the main sequence. The metals evident in a polluted WD's photosphere are most probably due to accreted debris - such as asteroids and minor planets that is continually being nudged inwards by a (thus far unseen) planet. To survive post-main-sequence evolution, the planet must have been in a radial location that ensured its protection from gravitational scattering, orbital expansion or engulfment and stellar mass-loss. Veras et al. put limits on that 'safe space' by combining the results of a multitude of published analytical and numerical models with their own calculations. They conclude that planets need to orbit their end-of-main-sequence host stars at 3-6 au (for 6-8 $M_{\odot}$ hosts) in order to persist until the WD phase. Minor planets are more susceptible to radiation effects (such as Yarkovsky, YORP) than gravitational ones, which puts them at risk from the variable luminosities of giantbranch stars, and they can also experience sublimation and rotational disruption due to interaction with the host star. For minor planets, the safe zone starts only at tens of au for more massive bodies, with smaller objects only being safe at thousands of au.

All in all, the message is somewhat positive: planets could exist around intermediate-mass stars, and we need to look closely at higher-mass WDs to spot the survivors.

\section{Paul Woods}

Published online: 4 March 2020

https://doi.org/10.1038/s41550-020-1056-9 